\title{
Molecular Typing of Staphylococcus aureus: Understanding and Controlling Epidemic Spread
}

\section{Sima Tokajian*}

Genomics and Proteomics Research Lab, Department of Biology, Lebanese American University, Byblos, Lebanon

\begin{abstract}
Methicillin resistant Staphylococcus aureus (MRSA) infections continue to spread worldwide. From an epidemiological perspective, risk factors for hospital acquired $(\mathrm{HA})$ infections are most associated, but not limited to with invasive medical devices, prolonged hospitalization, and surgical procedures. Strains causing infections in patients without risk factors for MRSA are known as community acquired (CA-MRSA). The line between HA- and CA-MRSA is blurring and clones of this pathogen are spreading across geographical borders due to international travel. Strain typing is an important component of epidemiological investigations that should be done to identify outbreak-related strains and hence to control new waves of MRSA infections both locally and internationally.
\end{abstract}

\section{Keywords: MRSA; CA-MRSA; ST80-MRSA-IV; Typing}

S. aureusis a well-recognized human pathogen of worldwide distribution. In Europe the most common CA-MRSA strain is CC80:ST80-IV $[1,2]$. In Denmark, CC80:ST80-IV was found to be the predominant cause of CA-MRSA infections in patients with family relationships in the Middle East. Studies conducted by Tokajian et al. in Lebanon [3] and Jordan [4] confirmed the notion that this clone was originally introduced in Denmark and possibly other parts of Europe from the Middle East. Hence, determining the disease burden, genotype, and clonal distribution are all important components that should be integrated in sound epidemiological investigations that should be implemented in developed and underdeveloped countries.

This requires routine strain typing to follow and identify outbreakrelated strains and to distinguish epidemic from endemic or sporadic isolates [5].This however, should be combined with the establishment of a sequence-based network in the Middle East to generate easily comparable typing data in electronic, portable form to be used by infection control units locally and internationally. This network could mimic the initiative of the currently available Seqnet.org which includes 44 laboratories from 25 European countries and one from Lebanon [6].

The most reliable typing methods with $S$. aureus are multilocus sequence typing (MLST) and pulse-Field gel electrophoresis (PFGE) [7]. MLST groups strains into sequence types (STs), and BURST (Based Upon Related Sequence Types) analysis is then used to group them into clonal complexes (CCs) $[8,9]$. PFGE has been found difficult to reproduce between laboratories and is limited in speed of analysis. MLST is usually preferred as a general typing technique because the data can be exchanged between different laboratories. However, major disadvantages of MLST are that it is expensive, laborious and time-consuming [8]. spa-typing of $S$. aureus is also an important sequence-based tool in the study of strain origin, clonal relatedness and epidemiology of $S$. aureus outbreaks. Protein A is a cell wall linked protein of $S$. aureus, and the X region of its gene (spa) consists of a variable number of direct repeats exhibiting an extensive polymorphism [10]. Sequencing of the repeat region generates informative typing results, hence allowing the grouping of the isolates into different spa types [11]. The BURP (Based Upon Repeat Pattern) algorithm is then used to group various spa-types into spa-CCs. spa-typing, unlike the others, can be used to study both the molecular evolution and hospital outbreaks of MRSA [8]. Sequencing of a single locus specific for $S$. aureus makes it the least tedious, expensive and time-consuming amongst the 3 methods. The technique's reliability on a single locus however, can be limiting with respect to discriminatory power due to same or related spa loci in different clonal lineages or to related repeat successions in different lineages [8].

There is an urgent need to standardize sampling regimen and typing of MRSA in the Middle East for epidemiological purposes. Real-time synchronization of typing data will help to: elucidate how strains that cause epidemics evolve, restrict CA-MRSA infections, and define the specific conditions that lead to the increased risk of spread of infections associated with foreign travel. Finally, a "search-and-destroy" policy as the one employed in Scandinavian countries should be implemented to ultimately restrict the effect of $S$. aureus infections.

\section{References}

1. Tristan A, Bes M, Meugnier H, Lina G, Bozdogan B, et al. (2007) Globa Distribution of Panton-Valentine Leukocidin-positive Methicillin-resistant Staphylococcus aureus, 2006. Emerg Infec Dis 13: 594-600.

2. Larsen AR, Böcher S, Stegger M, Goering R, Pallesen LV, et al. (2004) Epidemiology of European Community-Associated Methicillin-Resistant Staphylococcus aureus Clonal Complex 80 Type IV Strains Isolated in Denmark from 1993 to 2004. J Clin Microbiol 46: 62-68.

3. Tokajian ST, Khalil PA, Jabbour D, Rizk M, Farah MJ, et al. (2010) Molecular characterization of Staphylococcus aureus in Lebanon. Epidemiol Infect 138 707-712.

4. WissamAbi Khalil, Hashwa F, AsemShihabi, Tokajian S. Methicillin-resistan Staphylococcus aureus ST80-IV clone in children from Jordan. Diag Microbio Infect Dis. in press

5. Arbeit D (1995) Laboratory procedures for the epidemiologic analysis of

*Corresponding author: Sima Tokajian, Genomics and Proteomics Research Lab, Department of Biology, Lebanese American University, Byblos, Lebanon, Tel: +9619547263 (ext 2861); Fax: +9619546262; E-mail: stokajian@lau.edu.lb

Received March 23, 2012; Accepted March 23, 2012; Published March 25, 2012

Citation: Tokajian S (2012) Molecular Typing of Staphylococcus aureus Understanding and Controlling Epidemic Spread. J Forensic Res 3:e104. doi:10.4172/2157-7145.1000e104

Copyright: (c) 2012 Tokajian S. This is an open-access article distributed unde the terms of the Creative Commons Attribution License, which permits unrestricted use, distribution, and reproduction in any medium, provided the original author and source are credited. 
Citation: Tokajian S (2012) Molecular Typing of Staphylococcus aureus: Understanding and Controlling Epidemic Spread. J Forensic Res 3:e104. doi:10.4172/2157-7145.1000e104

Page 2 of 2

microorganisms. In: Murray R, baron J, Pfaller A, Tenover C, Yolken $\mathrm{H}$, (edn) Manual of Clinical Microbiology. (6 $6^{\text {th }}$ ed) American Society of Microbiology, Washington, DC 190-208.

6. Friedrich A, Witte W, Lencastre H, Hryniewicz, Scheres J, et al.(2008) A European laboratory networks for sequence-based typing typing of methicillinresistant Staphylococcus aureus (MRSA) as a communication platform between human and veterinary medicine-an update on SeqNet.org. Euro Surveill13: 1-5.

7. Aires de Sousa M, de Lencastre H (2004) Bridges from hospitals to the laboratory: genetic portraits of methicillin-resistant Staphylococcus aureus clones. FEMS Immunol Med Microbiol 40:101-111.

8. Deurenberg RH, Stobberingh EE (2008) The evolution of Staphylococcus aureus. Infect Genet Evol 8: 747-763.
9. Grundmann H, Hori S, Enright MC, Webster C, Tami A, et al. (2002) Determining the genetic structure of the natural population of Staphylococcus aureus: a comparison of multilocus sequence typing with pulsed-field ge electrophoresis, randomly amplified polymorphic DNA analysis, and phage typing. J Clin Microbiol 40: 4544-4546.

10. Brígido Mde $M$, Barardi $C R$, Bonjardin $C A$, Santos $C L$, Junqueira $M L$, et al.(1991) Nucleotide sequence of a variant prorein A of Staphylococcus aureus suggests molecular heterogenity among strains. J Basic Microbiol 5: 337-345.

11. Mellmann A, Weniger T, Berssenbrügge $C$, Keckevoet $U$, Friedrich AW, et a (2008) Characterization of the clonal relatedness among natural population of Staphylococcus aureus using spa typing and the BURP clustering. J Clin Microbiol 46: 2805-2808. 